Chemical Physics 126 (1988) 405-416

North-Holland, Amsterdam

\title{
PICOSECOND SPECTROSCOPY OF ELECTRONICALLY EXCITED SINGLET STATES IN BIPHENYLENE
}

\author{
T. ELSAESSER, F. LÄRMER, W. KAISER \\ Physik Department E 11, Technische Universität München, Arcisstrasse 21, D-8000 Munich, FRG
}

\author{
B. DICK \\ Max-Planck-Institut für Biophysikalische Chemie, Abt. Laserphysik, Am Fassberg, D-3400 Göttingen. FRG \\ M. NIEMEYER and W. LÜTTKE \\ Institut für Organische Chemie, Universität Göttingen, Tammannstrasse 2, D-3400 Göttingen, FRG
}

Received 20 August 1987 ; in final form 7 July 1988

\begin{abstract}
The photophysical properties of biphenylene- $h_{8}$ and biphenylene- $d_{8}$ in solution at room temperature are investigated with picosecond absorption and fluorescence spectroscopy. The weak fluorescence with complex vibrational structure originates entirely from the $S_{1}$ state. It decays monoexponentially with a lifetime of $240 \pm 20 \mathrm{ps}$. Upon photoexcitation to $S_{2}$ no emission attributable to the $S_{2}$ state was observed. Lifetime and quantum yield of fluorescence $\left(\Phi_{\mathrm{F}}=2.3 \times 10^{-4}\right)$ are the same for biphenylene- $h_{8}$ and for the completely deuterated compound, indicating that $\mathrm{C}-\mathrm{H}$ vibrations are not involved in the major radiationless decay process of the $S_{1}$ level, i.c. internal conversion $\left(\Phi_{1 C}>0.99\right)$. This conclusion is supported by INDO/S CI calculations where strong changes of the $\mathrm{CC}$ bond orders (particularly in the central $\mathrm{C}_{4}$ ring) with $\mathrm{S}_{0} \rightarrow \mathrm{S}_{1}$ excitation are found. The time-resolved excited-state absorption, measured at several wavelengths, decays biexponentially with time constants of $8 \pm 3$ and $250 \pm 40$ ps. Various interpretations of the 8 ps lifetime are discussed; the conclusion results that the 8 ps transient has to be assigned to the lifetime of the $S_{2}$ state even though the emission expected for an allowed transition with such a long lifetime is not observed. Strong $S_{1} \rightarrow S_{n}$ and $\mathrm{S}_{2} \rightarrow \mathrm{S}_{n}$ excited-state absorption is found as predicted by INDO/S calculations.
\end{abstract}

\section{Introduction}

Hückel's rule states that molecules with planar and rigid cyclic $\pi$-electron systems containing $(4 n+2) \pi$ electrons belong to the class of aromatic molecules whose prototype is benzene $(n=1)$ (I) [1]. Delocalization of the $\pi$ electrons in these aromatic molecules stabilizes them with respect to a hypothetical polyene structure with localized single and double bonds. On the other hand, systems containing $4 n \pi$ electrons in their perimeter would be destabilized by delocalization and hence prefer a localized arrangement of the $\pi$ electrons; these compounds are called antiaromatic (for a review, see ref. [2]). Whereas the investigations of the photophysical properties of aromatic molecules are legion [ 3,4$]$, only little is known about the photophysics of antiaromatic molecules. Nevertheless, the data available provide evidence that antiaromatic hydrocarbons behave drastically different from their aromatic counterparts. Wirz [5] has convincingly interpreted the particular behavior of the antiaromatic $4 n$-annulenes as a consequence of an avoided crossing between the potential surfaces of their $S_{0}$ and $S_{1}$ electronic states along the coordinate which relates the two possible bond-alternating structures and the intermediate delocalized structure of these molecules. Consequences of this fact are: (i) a long vibrational progression in the absorption and fluorescence spectra, (ii) the absence of mirror symmetry between the absorption and fluorescence spectra, and (iii) a large rate for radiationless deactivation of the $S_{1}$ state by internal conversion.

These photophysical properties should appear most clearly with cyclobutadiene (V) (see structures in scheme 1), the prototype of antiaromatic hydrocarbons. This compound is extremely unstable, having 


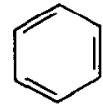

(I)

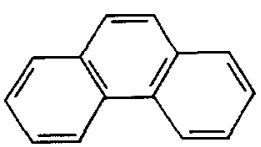

(III)<smiles>c1ccc2ccccc2c1</smiles>

(II)

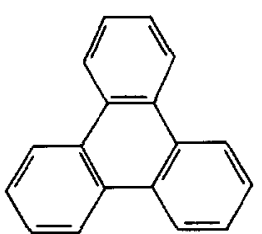

(IV)

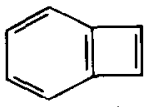

(V1)

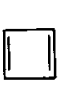

(v)

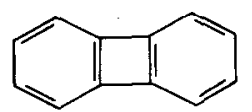

(VII)
Scheme 1. (I)-(IV) aromatic hydrocarbons, (V)-(VII) antiaromatic hydrocarbons.

been isolated only in rare-gas matrices at low temperatures [6-11]. It can be stabilized either sterically by substituting the four hydrogen atoms by tertiary butyl groups [12] or by annelation with one or two benzene rings, leading to benzocylcobutadiene (VI) and biphenylene (VII) [13]. These two molecules can also be regarded as antiaromatic $8 \pi$-electron or $12 \pi$ electron systems, respectively.

We have chosen biphenylene as example for our first investigation of antiaromatic molecules since its electronic spectrum is well understood: Shortly after the first synthesis of biphenylene [14], its absorption spectrum was studied in hexane solution [15] up to $48000 \mathrm{~cm}^{-1}$. It displays a weak band $\left(\epsilon=148 \mathrm{M}^{-1}\right.$ $\mathrm{cm}^{-1}$ ) with vibrational structure between 25000 and $27000 \mathrm{~cm}^{-1}$ partly overlapping with a second stronger band with maximum at $27910 \mathrm{~cm}^{-1}\left(\epsilon \approx 10^{4} \mathrm{M}^{-1}\right.$ $\mathrm{cm}^{-1}$ ). A very strong third band system is found with maximum at $40250 \mathrm{~cm}^{-1}\left(\epsilon \approx 10^{5} \mathrm{M}^{-1} \mathrm{~cm}^{-1}\right)$ having a shoulder at approximately $38000 \mathrm{~cm}^{-1}$. Analysis of the vibrational structure of the first absorption band in the vapor phase and in rigid EPA glass by Hochstrasser [16] indicated the forbidden nature of this transition and led to the assignment of $B_{1 \mathrm{~g}}$ symmetry for the $S_{1}$ state. With polarized absorption spectra of biphenylene in naphthalene host crystals [17], Hochstrasser showed that the second transition at $27910 \mathrm{~cm}^{-1}$ is long-axis polarized, and hence the $S_{2}$ state has $B_{3 u}$ symmetry in our axis convention. Hoshi et al. [18] measured the polarized absorption spectra of biphenylene in stretched polymer films and found that the transition at $38000 \mathrm{~cm}^{-1}$ is long-axis polarized $\left(B_{3 u}\right)$, whereas the transition at $40250 \mathrm{~cm}^{-1}$ is short-axis polarized $\left(B_{2 u}\right)$; they supported their argument additionally by PPP calculations including a configuration analysis. Recently these results were confirmed by studies of the linear dichroism and of the magnetic circular dichroism [19].

The fluorescence quantum yield of biphenylene is very low. Early observations of different emissions assigned to $S_{1} \rightarrow S_{0}$ and $S_{2} \rightarrow S_{0}$ fluorescence [20,21] were later shown to originate from impurities [22]. Shizuka et al. [23] were the first to observe the genuine fluorescence of biphenylene, measuring a quantum yield of $\Phi_{\mathrm{F}}=1.8 \times 10^{-4}$; they assigned this fluorescence to the $S_{2} \rightarrow S_{0}$ transition. This assignment was subsequently corrected to an $S_{1} \rightarrow S_{0}$ fluorescence after analysis of the vapor phase spectra also by Shizuka et al. [24]. Fluorescence from higher excited singlet states following two- and three-photon excitation has been reported by Lin and Topp [25], but a genuine $S_{2} \rightarrow S_{0}$ fluorescence has not been observed up to now.

The lifetimes of electronically excited states of biphenylene have been studied with time-resolved spectroscopy in the picosecond and microsecond range [25-27]. Upon excitation to $S_{1}$, a ground-state repopulation time of 10 ps was estimated in ref. [26 ]; this work however has not been confirmed. More recently, Lin and Topp [25] investigated the $S_{n} \rightarrow S_{0}$ fluorescence of biphenylene, resulting from two-photon excitation $\mathrm{S}_{0} \rightarrow \mathrm{S}_{1} \rightarrow \mathrm{S}_{n}$; from the dependence of the $S_{n}$ fluorescence intensity on the delay of the second excitation pulse they obtained an $S_{1}$ lifetime of $240 \pm 20$ ps. Phosphorescence of biphenylene has not been detected, indicating that the rate constant of internal conversion is much larger than the rate constant of intersystem crossing. A value of $\Phi_{\text {ISC }}<10^{-2}$ was estimated [25,27]. A population of the triplet state sufficient for transient absorption studies could only be achieved by flash photolysis of a mixture of biphenylene and naphthalene as a triplet sensitizer in cyclohexane solution. Thus a $T_{1}$ lifetime of $100 \mu \mathrm{s}$ 
was measured [27] which leads to an estimate of $10^{-6}$ for the phosphorescence yield of biphenylene.

Quantum-mechanical calculations of the electronic states of biphenylene have been performed several times $[18,19,28]$, mostly within the $\pi$-electron approximation. However, early attempts utilizing different parameterization schemes, optimized for polyenes and polyacenes, agreed only in the assignment of $B_{1 \mathrm{~g}}$ symmetry for the lowest excited state. With respect to the next few states they disagreed with each other and with the experimental results. The first calculation yielding the correct order and approximate transition energies for the lowest excited states was performed by Peradejordi et al. [28]. Their PPP. type calculation included doubly excited configurations which are now recognized as being necessary for the correct description of correlation effects, particularly in covalent states [29]. However, different sets of parameters were used for the carbon atoms of the four-membered ring and the other carbon atoms. This indicates that biphenylene behaves differently from aromatic molecules, mainly due to its four-membered ring which constitutes the particular antiaromatic character. The relaxation of the $\sigma$-bonding electrons upon excitation of the $\pi$ electrons will be affected by the high strain in this ring. Also, the small bond angle increases non-next-neighbour interactions which give rise to deviations from pairing symmetry [ 30 ]. Both effects can be accounted for by allvalence electron methods, and applications to biphenylene have been reported [19,30-32]. However, as far as we know no calculations of energies and oscillator strengths of the $S_{1} \rightarrow S_{n}$ and $S_{2} \rightarrow S_{n}$ transitions were performed.

In this paper, we present a systematic investigation of the picosecond photophysics of biphenylene in liquid solution. The fluorescence kinetics is measured directly and compared to transient absorption changes which are observed in picosecond pump and probe experiments. In this way information on the radiationless processes is obtained.

To support the interpretation of the experimental data, INDO/S CI calculations on biphenylene and, for comparison, the aromatic hydrocarbons benzene and triphenylene were performed.

\section{Experimental}

Biphenylene- $h_{8}$ and biphenylene- $d_{8}$ were synthesized according to refs. [33] and [34], respectively. After purification of both substances by preparative gas chromatography (GLC: $3 \mathrm{~m} \times \frac{1}{4}$ " all glass system, $15 \%$ OV 101 on Chromosorb W AW/DMCS 60/80) their purity was checked by comparison of absorption spectra with the fluorescence excitation spectra. The ovcrall deutcrium content of biphenylene- $d_{8}$ (determined by mass spectroscopy) was $96 \% ; 77 \%$ of the molecules were found to be perdeuterated. We used spectrograde cyclohexane (Merck Uvasol) as solvent to prepare solutions with concentrations of $10^{-5}$ to $10^{-3} \mathrm{M}$ for absorption and fluorescence spectroscopy, and $3 \times 10^{-3} \mathrm{M}$ for picosecond experiments.

The spectra were obtained with a Cary 219 absorption spectrometer and a Perkin-Elmer MPF 44B fluorimeter, both computer controlled; the fluorescence measurements were corrected for the spectral sensitivity of the apparatus. The fluorescence quantum yield was measured with reference to 9,10-diphenylanthracene (DPA) in cyclohexane $\left(\Phi_{\mathrm{F}}=0.95\right.$ [35]). Because of the great difference of the emission intensities, a direct comparison of biphenylene and DPA was not practicable. Therefore, the fluorescence of DPA was attenuated with a neutral-density filter (Schott NG4, $4 \mathrm{~mm}$ ), and the attenuation factor of the filter was determined in a separate experiment with lower excitation intensity.

Single picosecond light pulses at $\tilde{\nu}=9398 \mathrm{~cm}^{-1}$ were generated in a Nd:YAG laser system consisting of a passively mode-locked oscillator, a single-pulse selector and a two-stage amplifier. Frequency tripling of these light pulses [36] yielded pulses at 28194 $\mathrm{cm}^{-1}$ of $21 \mathrm{ps}$ duration which were used to excite the sample. The decay of the excited states was monitored through fluorescence and transient-absorption measurements. Fluorescence decay curves were recorded with a Hadland Photonics streak-camera with a time resolution of $4 \mathrm{ps}$. The time dependence of the excited-state absorption was measured with a weak probe pulse of variable time delay relative to the pump pulse. Probe pulses at different wavelengths were generated by nonlinear frequency mixing of pulses from the Nd:YAG laser with pulses from a tunable travelling-wave dye laser emitting in the near 
infrared [37]. Pulses at $19600 \mathrm{~cm}^{-1}(510 \mathrm{~nm})$ and $18180 \mathrm{~cm}^{-1}(550 \mathrm{~nm})$ were generated with a singlecell travelling-wave dye laser [38]. The cross-correlation of the pump and probe pulses gives the time resolution of the apparatus and marks the point of zero time-delay [39]. We performed control measurements of ground-state bleaching and stimulated emission of laser dyes with known $S_{1}$ lifetimes in the nanosecond range. Here the signal follows the integral over the correlation function [40]. In this way the time resolution of the apparatus was determined to be $4 \mathrm{ps}$. The polarization direction of the probe light pulses was set $54.7^{\circ}$ to that of the pump pulse. In this "magic angle" setup rotational relaxation does not contribute to the absorption transients.

\section{Results}

\subsection{Steady-state absorption and fluorescence spectra}

Fig. 1 displays the absorption and fluorescence spectra of biphenylene. The Stokes shift between both spectra is $4700 \mathrm{~cm}^{-1}$. The fluorescence spectrum shows a complex vibrational structure with intervals between successive maxima of $350-400 \mathrm{~cm}^{-1}$. A very low quantum yield of $\Phi_{\mathrm{F}}=2.3 \times 10^{-4}$ was measured; this is consistent with earlier measurements: ref. [23]: $\Phi_{\mathrm{F}}=(1.8 \pm 0.3) \times 10^{-4}\left(\lambda_{\mathrm{exc}}=350 \mathrm{~nm}\right.$, solutions in cyclohexane, ethanol or acetonitrile, $20^{\circ} \mathrm{C}$ ); ref. [24]: $\Phi_{\mathrm{F}}=3.3 \times 10^{-4}\left(\lambda_{\text {exc }}=382 \mathrm{~nm}\right.$, vapour, $\left.90^{\circ} \mathrm{C}\right)$. It is important to note that neither the fluorescence spectrum nor the fluorescence quantum yield depend on the excitation frequency. The spectrum shown here was obtained by irradiation into the $S_{0} \rightarrow S_{2}$ absorption band $\left(28200 \mathrm{~cm}^{-1}\right)$, but irradiation into the $S_{0} \rightarrow S_{1}$ absorption band $\left(25000 \mathrm{~cm}^{-1}\right)$ leads to exactly the same spectrum. Therefore all the fluorescence originates from the $S_{1}$ state [18]. No emission attributable to the $S_{2}$ state could be observed.

From the spectra in fig. 1 the radiative lifetime $\tau_{\text {rad }}$ of the $S_{1}$ state can be estimated utilizing the Strickler-Berg relation [41]. We find a value of $\tau_{\text {rad }}=2.5 \mu$ s for this forbidden transition. The fluorescence quantum yield and the lifetime of the $S_{1}$ state of $250 \mathrm{ps}$ (see below) give a value of $\tau_{\mathrm{rad}}=1.1 \mu \mathrm{s}$. This discrepancy is not surprising because of the partial overlap of the $S_{0} \rightarrow S_{1}$ band with the neighbouring

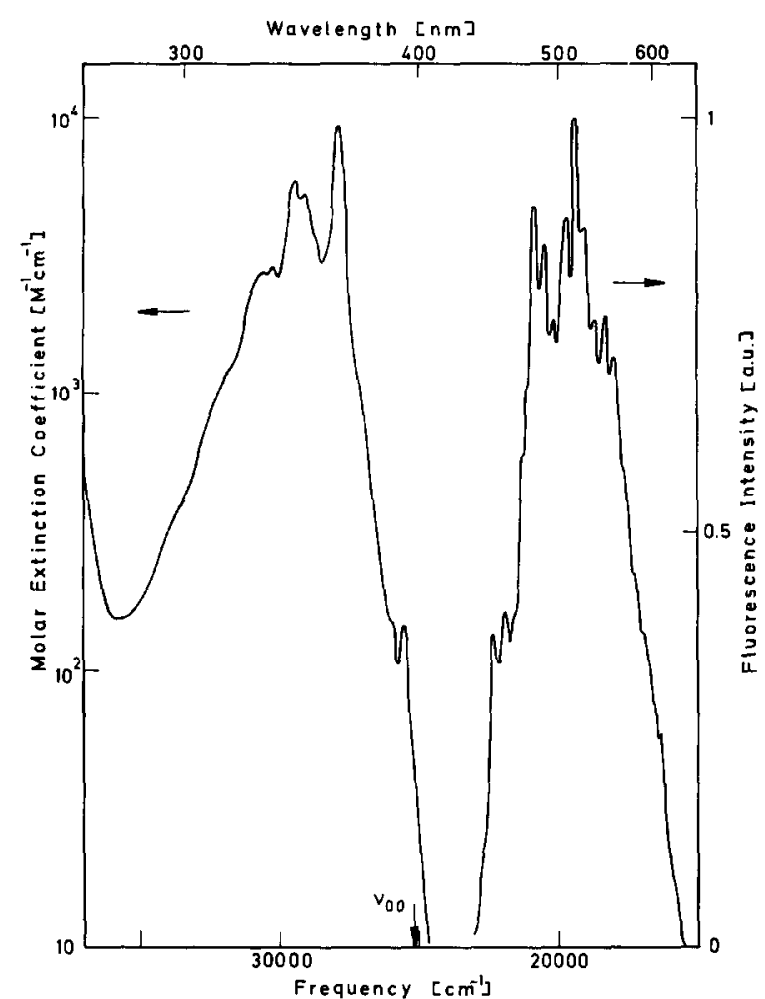

Fig. 1. Absorption and fluorescence spectra of biphenylene in cyclohexane solution. (Left: molar extinction coefficient, logarithmic scale; right: fluorescence intensity, linear scale.)

$S_{0} \rightarrow S_{2}$ band. Moreover, the Strickler-Berg relation is based on the assumption that the equilibrium geometry in $S_{0}$ and $S_{1}$ is the same. However, as emphasized in refs. [22,24], the molecular geometry of biphenylene changes considerably on excitation as is characteristic for antiaromatic molecules [5].

\subsection{Transient absorption and fluorescence decay measurements}

The decay kinetics of the very weak fluorescence of biphenylene is plotted in fig. 2 (points). The sample was excited by a single picosecond pulse at 28194 $\mathrm{cm}^{-1}$ in the $S_{0} \rightarrow S_{2}$ transition. The decay of the spectrally integrated emission was monitored directly by the streak camera. The solid line in fig. 2 , which matches well the experimental points, represents a 


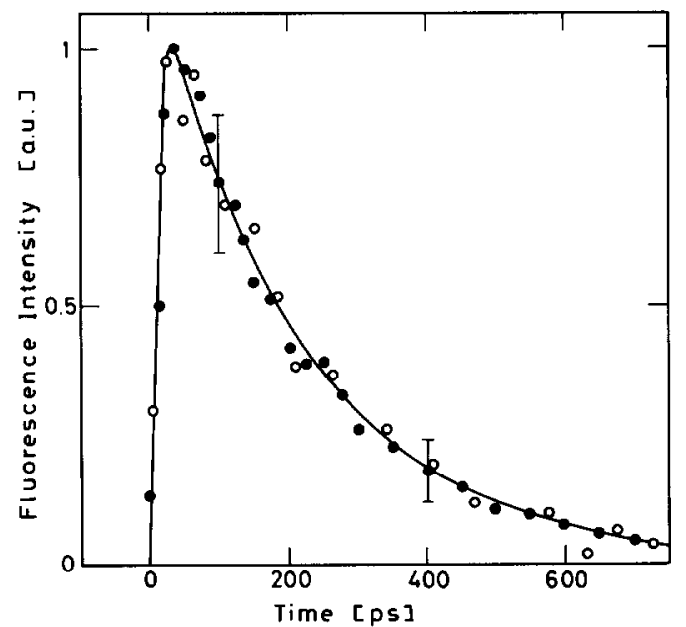

Fig. 2. Streak-camera traces of the fluorescence decay of biphenylene- $h_{8}$ (points) and biphenylene- $d_{8}$ (circles) after picosecond excitation at $28200 \mathrm{~cm}^{-1}$. Solid line: theoretical fit with time constant of $250 \mathrm{ps}$.

monoexponential decay curve calculated with a time constant of $250 \mathrm{ps} \#$.

The results of the transient absorption measurements are presented in fig. 3. The picosecond pump pulse at $28194 \mathrm{~cm}^{-1}$ excites approximately $10 \%$ of the molecules to the $S_{2}$ state. Strong transient excited-state absorption was found in the range between $27780 \mathrm{~cm}^{-1}(360 \mathrm{~nm})$ and $21740 \mathrm{~cm}^{-1}(460$ $\mathrm{nm})$ and in the range from $18800 \mathrm{~cm}^{-1}(532 \mathrm{~nm})$ to $18180 \mathrm{~cm}^{-1}(550 \mathrm{~nm})$. The extinction coefficient of the excited-state absorption exceeds that of the ground-state absorption at all frequencies investigated. Consequently the ground-state recovery time cannot be measured by a pump and probe experiment in this region of the absorption spectrum.

Fig. 3 presents the time dependence of the transient absorption at three selected frequencies: At $26315 \mathrm{~cm}^{-1}(360 \mathrm{~nm})$ in the range of the $S_{0} \rightarrow S_{1}$ absorption, at $18796 \mathrm{~cm}^{-1}(532 \mathrm{~nm})$ in the range of the fluorescence spectrum, and in between at 22940 $\mathrm{cm}^{-1}(436 \mathrm{~nm})$. The change of the absorbance $\Delta A=\log \left(T_{0} / T\right)$ is plotted as a function of the delay time between the pump pulse and the probe pulse; $T_{0}$

\#1 The zero point of the time scale in fig. 3 is arbitrarily chosen to coincide with the rising part of the streak curve; the exact relation between the excitation pulse and the onset of the emission is uncertain within approximately $10 \mathrm{ps}$ because of the triggering jitter of the streak camera.

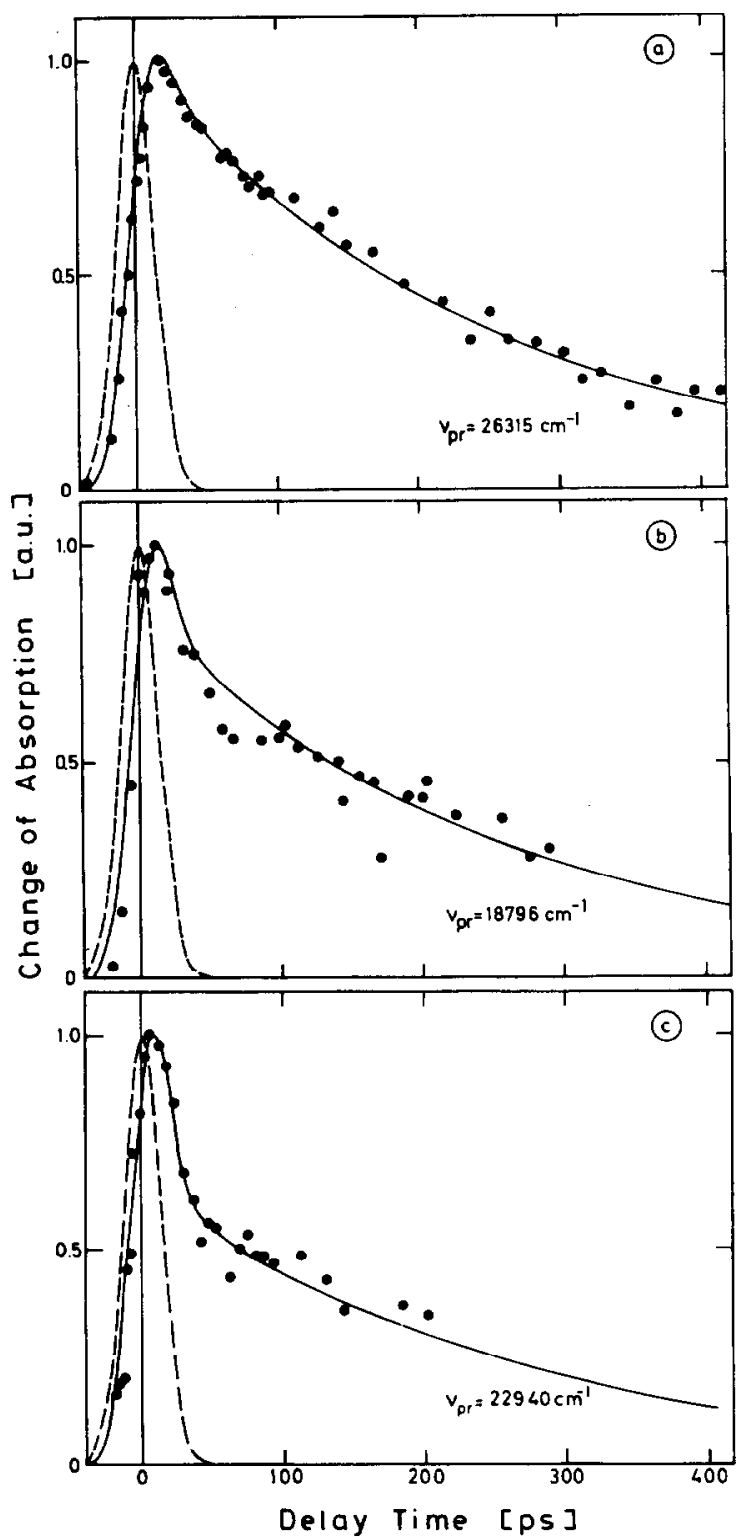

Fig. 3. Transient excited-state absorption of biphenylene at (a) $26315 \mathrm{~cm}^{-1}(380 \mathrm{~nm})$, (b) $18796 \mathrm{~cm}^{-1}(532 \mathrm{~nm})$ and (c) 22940 $\mathrm{cm}^{-1}(436 \mathrm{~nm}$ ). The measured signal (points) is plotted versus the delay time between the pump pulse at $28200 \mathrm{~cm}^{-1}(354 \mathrm{~nm})$ and the probe pulse. Solid line: theoretical fit with time constants of 8 ps and 250 ps for the fast and slow decay component, respectively. Dashed line: cross correlation of the pump and probe pulses indicating the temporal resolution of the apparatus.

and $T$ are the transmissions of the sample at the frequency of the probe pulse before and after excitation, respectively. The absorbance change rises within the 
time resolution of the experiment. The latter is determined by the cross correlation of the pump pulses and probe pulses shown as dashed line in fig. 3. At all probe frequencies, the decay of the transient absorption is not monoexponential but consists of a first fast and a subsequent slower contribution. The fast component is especially strong at the probing frequency of $22940 \mathrm{~cm}^{-1}(436 \mathrm{~nm})$ as shown in fig. 3c. From this measurement the time constant of the fast component is obtained with good accuracy.

The solid lines in fig. 3 represent the fit of a sum of two exponentials to the experimental data points. In all cases the two time constants were $8 \pm 3$ ps for the fast component and $240 \pm 40$ ps for the slow component.

The excited-state extinction coefficients $\epsilon_{1}$ and $\epsilon_{2}$ corresponding to the slow and to the fast signal component, respectively, are estimated from the absolute value of the measured signal at $26315 \mathrm{~cm}^{-1}(380 \mathrm{~nm})$ and $22940 \mathrm{~cm}^{-1}(436 \mathrm{~nm})$. At these frequencies the competing contributions of ground state bleaching and stimulated emission are negligible. The experimental result that the fluorescence quantum yield is independent of the excitation wavelength [24] suggests that sequential population takes place. This kinetic behavior enables us to obtain values of $\epsilon_{1} \approx 10^{4}$ $\mathrm{M}^{-1} \mathrm{~cm}^{-1}$ and $\epsilon_{2} \approx 2 \times 10^{4} \mathrm{M}^{-1} \mathrm{~cm}^{-1}$ at $380 \mathrm{~nm}$ and $\epsilon_{1} \approx 5 \times 10^{3} \mathrm{M}^{-1} \mathrm{~cm}^{-1}$ and $\epsilon_{2} \approx 2.5 \times 10^{4} \mathrm{M}^{-1} \mathrm{~cm}^{-1}$ at $436 \mathrm{~nm}$. Both excited state extinction coefficients are larger than those of the $S_{0} \rightarrow S_{1}$ and the $S_{0} \rightarrow S_{2}$ absorption.

An interesting observation was made at a probing frequency of $19600 \mathrm{~cm}^{-1}(510 \mathrm{~nm})$. A very small gain of the probing light was detected which rose with a delay of approximately 10-20 ps following the excitation pulse. At this frequency the cross section for stimulated emission is slightly larger than the excited-state absorption cross section.

\section{3. $H / D$ isotope effect}

In order to study the contribution of the $\mathrm{CH}$ vibrations as accepting modes of the internal conversion of $S_{1}$ to $S_{0}$, we also measured the quantum yield $\Phi_{F}$ and the decay time $\tau_{1}$ of the fluorescence of perdeuterated biphenylene, $C_{12} D_{8}$. To determine $\Phi_{F}$ of $\mathrm{C}_{12} \mathrm{D}_{8}$, the fluorescence of equally concentrated solutions of $\mathrm{C}_{12} \mathrm{D}_{8}$ and $\mathrm{C}_{12} \mathrm{H}_{8}$ in cyclohexane were compared. No intensity difference was observed, the signal-to-noise ratio being 200:1. The decay of the fluorescence of $\mathrm{C}_{12} \mathrm{D}_{8}$, mcasured with the strcak camera, is shown in fig. 3 (open circles); a monoexponential decay with a time constant of $250 \pm 30$ ps is found (i.e. identical with that of $\mathrm{C}_{12} \mathrm{H}_{8}$ ). This lack of an $\mathrm{H} / \mathrm{D}$ isotope effect on the internal conversion strongly supports the conclusion that in biphenylene the deactivation of the $S_{1}$ excited molecule is predominantly caused by vibrations of the carbon skeleton and not of the $\mathrm{CH}$-bonds.

\subsection{INDO/S calculations}

The energies, oscillator strengths, symmetries, and the bond orders of the lower singlet states of biphenylene were calculated by the all-valence-electron INDO/S method, including 200 energy-selected singly and doubly excited configurations. Coulomb integrals were calculated with the Pariser formula which gives better results than the Nishimoto-Mataga formula when doubly excited configurations are included [42]. All parameters were taken from the standard CNDO/S procedure [43] with the exception of $\beta_{C}$ which was set to $-16.0 \mathrm{eV}$ [32]. The data are collected in tables 1 and 2 . The four experimentally assigned $S_{0} \rightarrow S_{n}$ transitions $[16,18,19]$ are reproduced in the correct order, and the calculated oscillator strengths are in good agreement with experimental data. As usual when doubly excited configurations are involved, the calculated excitation energies are too large by $2000-4000 \mathrm{~cm}^{-1}$. This observation can be attributed to the fact that the doubly excited configurations yield contributions to the correlation energy for the ground state which are only accounted for by triply excited configurations for the excited states. The energy differences and oscillator strengths for transitions between excited states should be predicted with a similar accuracy. However, a source of error not accounted for by our calculation is the unknown but remarkable change in equilibrium geometry in the $S_{1}$ state compared to the $S_{0}$ state, as was emphasized in refs. [22,24].

The calculated patterns for the $S_{0} \rightarrow S_{n}, S_{1} \rightarrow S_{n}$, and $S_{2} \rightarrow S_{n}$ oscillator strengths are shown in fig. 4 . Transitions with $f<0.001$ are displayed as short bars. Polarization directions are indicated by $L, M$, and $S$ for the long, the medium, and the short (out-of-plane) 
Table 1

Spectroscopic data for the lowest excited singlet states $\mathrm{S}_{n}$ of biphenylene calculated with the INDO/S method. $E$ : transition energy from the ground state $S_{0}$ in units of $1000 \mathrm{~cm}^{-1}, f$ : oscillator strength, \%D: contribution of doubly excited configurations (in percent of the norm ) to the wavefunction. Polarization-directions of the transition moment vectors are indicated by $L$ and $M$ for the long and medium axis of the molecule. The transition energies and molar extinction coefficients for the lowest four experimentally verified transitions are shown for comparison

\begin{tabular}{|c|c|c|c|c|c|c|c|}
\hline \multicolumn{6}{|c|}{ Calculated } & \multicolumn{2}{|c|}{ Experimental } \\
\hline$n$ & $E$ & $f$ & pol. & $\% \mathrm{D}$ & sym. & $E$ & $\log \epsilon$ \\
\hline 0 & 0.0 & & & 3.2 & $A_{g}$ & & \\
\hline 1 & 27.6 & & & 3.7 & $B_{18}^{6}$ & 25.5 & 2.0 \\
\hline 2 & 30.9 & 0.094 & $\mathrm{~L}$ & 4.1 & $B_{3 u}$ & 27.9 & 4.0 \\
\hline 3 & 36.1 & & & 5.6 & $A_{B}$ & & \\
\hline 4 & 41.9 & 0.034 & $\mathbf{M}$ & 5.2 & $\mathrm{~B}_{2 \mathrm{u}}$ & 38.0 & 3.0 \\
\hline 5 & 43.0 & & & 1.6 & $B_{3 g}$ & & \\
\hline 6 & 43.9 & 1.248 & L & 3.3 & $B_{3 u}$ & 40.2 & 5.0 \\
\hline 7 & 45.2 & & & 6.7 & $B_{18}$ & & \\
\hline 8 & 46.7 & & & 88.8 & $A_{g}$ & & \\
\hline 9 & 46.8 & & & 0.4 & $\mathrm{~A}_{\mathrm{u}}$ & & \\
\hline 10 & 48.2 & & & 33.4 & $A_{g}$ & & \\
\hline 11 & 48.4 & 0.070 & M & 27.1 & $\mathbf{B}_{2 \mathbf{u}}$ & & \\
\hline
\end{tabular}

axis of the molecule, respectively. For comparison with the experimental data we can distinguish two main regions of interest: The range from 18000 to $23000 \mathrm{~cm}^{-1}$, where no ground-state absorption exists, and the range from 26000 to $28000 \mathrm{~cm}^{-1}$. In our calculation these regions correspond to transitions from $S_{1}$ or $S_{2}$ to very highly excited states of biphenylene in the range $S_{8}$ to $S_{30}$. The average distance of these highly excited states is approximately $550 \mathrm{~cm}^{-1}$, probably much less than the homogeneous width of these states or the energies of some of the vibrational modes in biphenylene. Hence one cannot expect that the Born-Oppenheimer approximation is applicable. Although only few of the calculated transitions in the $\mathbf{S}_{1} \rightarrow \mathbf{S}_{n}$ and $\mathbf{S}_{2} \rightarrow \mathbf{S}_{n}$ spectra have oscillator strengths greater than 0.01 , all the other states will be accessible by optical transitions due to the non-BornOppenheimer interaction.

In the first region, 18000 to $23000 \mathrm{~cm}^{-1}$, the INDO/S calculation predicts a long-axis-polarized transition in both excited-state spectra. For the $S_{1} \rightarrow S_{n}$ spectrum it has an oscillator strength $f=0.08$, whereas for the $\mathbf{S}_{2} \rightarrow \mathbf{S}_{n}$ spectrum $f=0.12$. In the second region, 26000 to $28000 \mathrm{~cm}^{-1}$, only a weak long-axis- polarized transition with $f=0.03$ is calculated for both excited-state spectra. Since the experimental data indicate a transition of similar strength to that in the first region, a further strong transition must be involved at least through vibronic coupling. In the $\mathrm{S}_{1} \rightarrow \mathrm{S}_{n}$ spectrum this could be the long-axis-polarized transition at $32500 \mathrm{~cm}^{-1}$ with $f=0.68$. The $S_{2} \rightarrow S_{n}$ spectrum has two close-lying transitions, at 31700 $\mathrm{cm}^{-1}(f=0.22)$ and $34200 \mathrm{~cm}^{-1} \quad(f=0.23)$, both long-axis polarized. In summary, the INDO/S calculation predicts very similar spectra for the $S_{1} \rightarrow S_{n}$ and $S_{2} \rightarrow S_{n}$ transitions of biphenylene in the range 18000 to $35000 \mathrm{~cm}^{-1}$, dominated by long-axis polarized transitions.

In order to estimate the change of geometry upon excitation we have calculated the bond orders for the carbon-carbon bonds from the INDO/S wavefunctions of the ground state and the two lowest excited states. The all-valence-electron bond order concept proposed by Cohen [44] was used within the basis of orthogonal atomic orbitals. This treatment gives equal weight to bonding and antibonding contributions. Under these conditions the Cohen bond order is a generalization of the Coulson $\pi$-bond order [45]. For the reference compounds ethane, ethylene, acetylene, and benzene these Cohen bond orders calculated from the INDO/S wavefunction of the ground state are $1.082,1.994,2.944$, and 1.689 .

The results of biphenylene (VII) and cyclobutadiene (V) and, for comparison, of two typical aromatic compounds, benzene (I) and triphenylene (IV), are collected in table 2 . For the ground state the total bond order $B_{\text {total }}$ and the out-of-plane $\pi$ component $B_{\pi}$ are shown. The latter corresponds to the bond order of PPP theories. For the excited states the change of the bond order $\triangle B=B\left(S_{n}\right)-B\left(S_{0}\right)$ is given. Since $\sigma \sigma^{*}$ excited configurations yield only a minute contribution to the wavefunctions of $S_{1}$ and $S_{2}$, the change in bond order is almost entirely due to the change in the out-of-plane $\pi$ component.

Bond orders correlate well with bond lengths. Hence an increase of a bond order upon excitation indicates that the corresponding bond will be stronger and shorter in the excited state. In the two aromatic examples, benzene and triphenylene, most bond orders change by less than 0.1 in $S_{1}$ and $S_{2}$. In triphenylene the largest change occurs for bond $b$, and this change is negative. The $S_{1}$ state of biphenylene and 
Table 2

INDO/S CI bond orders $B$ of antiaromatic and aromatic hydrocarbons

\begin{tabular}{|c|c|c|c|c|c|}
\hline \multirow[t]{2}{*}{ Substance } & \multirow[t]{2}{*}{ Bond } & \multicolumn{2}{|l|}{$S_{0}$} & \multirow{2}{*}{$\begin{array}{l}\mathrm{S}_{1} \\
\Delta B_{\text {total }}\end{array}$} & \multirow{2}{*}{$\begin{array}{l}\mathrm{S}_{2} \\
\Delta B_{\text {total }}\end{array}$} \\
\hline & & $B_{\text {total }}$ & $B_{\pi}$ & & \\
\hline biphenylene (VII) & $\begin{array}{l}a \\
b \\
c \\
d \\
e\end{array}$ & $\begin{array}{l}1.177 \\
1.503 \\
1.787 \\
1.596 \\
1.785\end{array}$ & $\begin{array}{l}0.200 \\
0.528 \\
0.736 \\
0.568 \\
0.738\end{array}$ & $\begin{array}{r}0.299 \\
-0.260 \\
-0.145 \\
0.120 \\
-0.190\end{array}$ & $\begin{array}{r}0.123 \\
-0.086 \\
-0.119 \\
-0.043 \\
-0.059\end{array}$ \\
\hline cyclobutadiene (V) & $\begin{array}{l}a \\
b\end{array}$ & $\begin{array}{l}1.135 \\
1.786\end{array}$ & $\begin{array}{l}0.152 \\
0.777\end{array}$ & $\begin{array}{r}0.542 \\
-0.648\end{array}$ & $\begin{array}{r}0.335 \\
-0.272\end{array}$ \\
\hline triphenylene (IV) & $\begin{array}{l}a \\
b \\
c \\
d \\
e\end{array}$ & $\begin{array}{l}1.405 \\
1.606 \\
1.627 \\
1.734 \\
1.663\end{array}$ & $\begin{array}{l}0.385 \\
0.588 \\
0.600 \\
0.699 \\
0.625\end{array}$ & $\begin{array}{r}0.061 \\
-0.110 \\
-0.045 \\
-0.036 \\
-0.031\end{array}$ & $\begin{array}{r}0.054 \\
-0.131 \\
-0.037 \\
-0.024 \\
-0.036\end{array}$ \\
\hline benzene (I) & (vil) & (v) & 0.650 & -0.178 & -0.156 \\
\hline
\end{tabular}

of cyclobutadiene yield a completely different picture: all bond orders change by more than 0.1 . The largest changes occur in the four-membered ring: the bond order of bond $b$, which in biphenylene is also part of the benzene ring, decreases by 0.26 , whereas the bond order of bond a increases by 0.3 . This almost reverses the bond orders for these two bonds. Hence a drastic shortening of bond a and lengthening of bond $b$ are expected to occur in $S_{1}$. The vibration associated with the corresponding normal coordinate should, therefore, be very effective in coupling $S_{0}$ to $S_{1}$. Interestingly, such strong changes of bond orders are not found in the $S_{2}$ state of biphenylene. In fact, it behaves quite similar to the $S_{1}$ and $S_{2}$ states of the aromatic reference compounds. It should be noted that the strong changes of $\mathrm{CC}$ bond orders in the central ring of biphenylene with the $S_{0} \rightarrow S_{1}$ excitation are very similar to those calculated for cyclobutadiene itself (see table 2).

\section{Discussion}

Excitation of biphenylene to $S_{2}$ produces two transient species with lifetimes of $8 \pm 3$ ps and $250 \pm 40$ ps, respectively. Whereas the slowly decaying species occurs in the fluorescence and the transient absorption measurements, the fast decaying species is seen only in the latter.

The decay time of 250 ps can safely be interpreted as the lifetime of the electronic state $S_{1}$ since it is the fluorescence decay time and all fluorescence originates from the $S_{1}$ state: the excitation of biphenylene to $S_{1}$ and $S_{2}$, respectively, yields identical fluorescence spectra.

The component in the transient absorption spectrum decaying with $250 \mathrm{ps}$ is, therefore, assigned to transient $\mathrm{S}_{1} \rightarrow \mathrm{S}_{n}$ absorption. This lifetime for the $\mathrm{S}_{1}$ state agrees well with the result found in ref. [25] by transient fluorescence excitation experiments. From this observed lifetime and the fluorescence quantum yield $\left(\Phi_{1}=2.3 \times 10^{-4}\right)$ a radiative lifetime of 


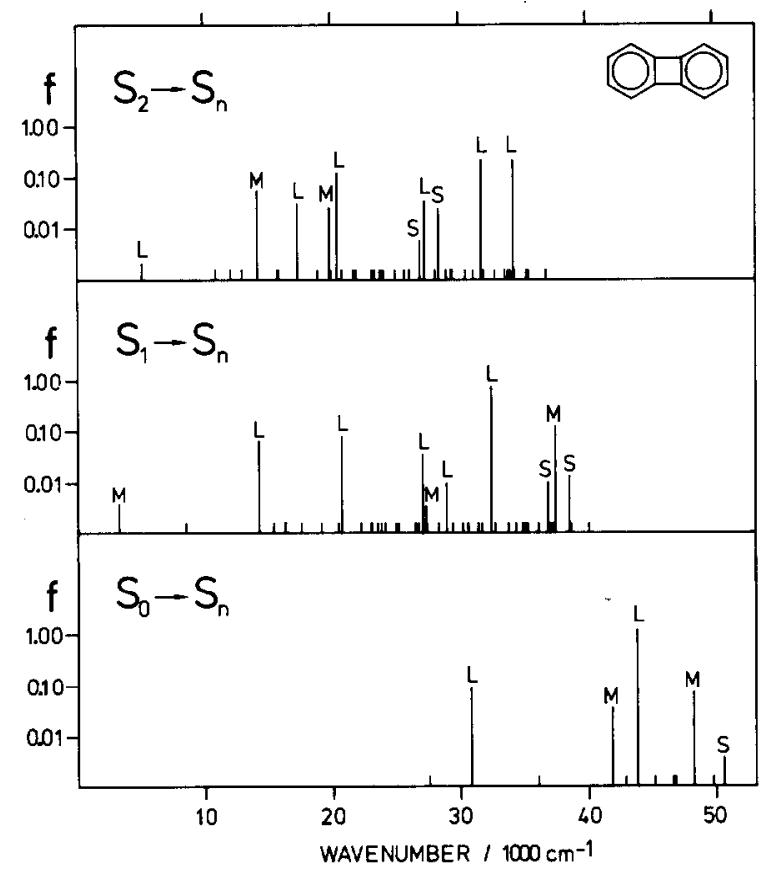

Fig. 4. Transition energies and oscillator strengths for the $\mathbf{S}_{0} \rightarrow \mathbf{S}_{n}$, $\mathrm{S}_{1} \rightarrow \mathrm{S}_{n}$, and $\mathrm{S}_{2} \rightarrow \mathrm{S}_{n}$ absorption bands of biphenylene by INDO/S calculation. Transitions with oscillator strengths $f<0.001$ are shown as small bars. The letters $\mathrm{L}, \mathrm{M}$ and $\mathrm{S}$ indicate that the corresponding transition is polarized along the long, medium, or short (out of plane) axis of the molecule.

$1.1 \pm 0.2 \mu \mathrm{s}$ is calculated. This long radiative lifetime is a consequence of the $B_{g}$ symmetry of the $S_{1}$ state which yields a very small transition dipole moment for the $S_{1} \rightarrow S_{0}$ transition.

In table 3 some photophysical parameters of the $S_{1}$ state of biphenylene are compared to those of two characteristic aromatic compounds, phenanthrene and triphenylene. In these two molecules the transitions between $S_{0}$ and $S_{1}$ are also of forbidden nature, though not by inversion symmetry but by pairing symmetry. Their radiative rate constant $k_{\mathrm{F}}$ is by a factor of 6 larger than that of biphenylene. The upper limit of $k_{\mathrm{ISC}}$ of biphenylene is close to the value of the two aromatic compounds. However, in biphenylene the rate constant of internal conversion, $k_{\mathrm{IC}}$, is larger by a factor of 3000 in comparison to the aromatic compounds.

Thus, in biphenylene internal conversion $S_{1} \rightarrow S_{0}$ is the predominant decay channel. The lack of an $H / D$ isotope effect on the $S_{1}$ lifetime shows, that the active vibration responsible for internal conversion is not a $\mathrm{C}-\mathrm{H}$ vibration, but rather a vibration of the carbon skeleton. This observation, together with the fact that $1-\left(\Phi_{\mathrm{F}}+\Phi_{\mathrm{ISC}}\right)>0.99$, is in contrast to the behaviour expected for aromatic molecules [46]. Hence it is likely that the vibration most active in internal conversion is related to the antiaromatic character of biphenylene, presumably associated with the normal coordinate along which the avoided crossing discussed by Wirz [5] occurs. This conclusion is supported by the calculated changes in the bond orders of the four-membered ring upon excitation $S_{0} \rightarrow S_{1}$ (table 2).

The interpretation of the fast component of the biexponential decay of the transient absorption is not straightforward. It cannot be due to a nonlinear effect because the relative amplitudes of the two components are independent of the energy of the pump pulse. An artifact of the apparatus can be excluded since with many other compounds investigated only

Table 3

Comparison of decay parameters of the $S_{1}$ state of biphenylene (VII), triphenylene (IV) and phenanthrene (III). $\tau_{1}$ : fluorescence lifetime; $\Phi_{\mathrm{F}}\left(k_{\mathrm{F}}\right), \Phi_{\mathrm{ISC}}\left(k_{\mathrm{ISC}}\right), \Phi_{\mathrm{IC}}\left(k_{\mathrm{IC}}\right)$ : quantum yields (rate constants) of fluorescence, intersystem crossing and internal conversion

\begin{tabular}{lccc}
\hline & Biphenylene & Triphenylene ${ }^{\text {a) }}$ & Phenanthrene $^{\text {a) }}$ \\
\hline$\tau_{\text {rad }}(\mathrm{ns})$ & 1100 & 250 & 460 \\
$\tau_{\mathrm{I}}(\mathrm{ns})$ & $0.250^{\mathrm{b})}$ & 36.6 & 59.5 \\
$\Phi_{\mathrm{F}}$ & $0.00023^{\mathrm{b})}$ & 0.07 & 0.13 \\
$\Phi_{\mathrm{ISC}}$ & $<0.01^{\mathrm{c}}$ & 0.86 & 0.80 \\
$\Phi_{\mathrm{IC}}$ & $>0.99$ & $<0.05$ & 0.07 \\
$k_{\mathrm{F}}\left(\mathrm{s}^{-1}\right)$ & $3.2 \times 10^{5}$ & $1.9 \times 10^{6}$ & $2.2 \times 10^{6}$ \\
$k_{\mathrm{ISC}}\left(\mathrm{s}^{-1}\right)$ & $<4.0 \times 10^{7}$ & $2.3 \times 10^{7}$ & $1.3 \times 10^{7}$ \\
$k_{\mathrm{IC}}\left(\mathrm{s}^{-1}\right)$ & $4.0 \times 10^{9}$ & $<1.4 \times 10^{6}$ & $1.2 \times 10^{6}$ \\
\hline
\end{tabular}

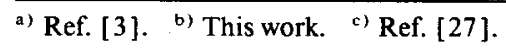


monoexponential decays were observed. In the following possible assignments for the $8 \mathrm{ps}$ transient of biphenylene are discussed.

(a) Concerning its magnitude, the $8 \mathrm{ps}$ transient could be interpreted as a vibrational relaxation time, either of an intramolecular redistribution (IVR) [4749 ] or of the dissipation of the vibrational energy into the solvent [50]. Vibrationally hot molecules show a strong increase of absorption intensity at the longwavelength edge of their absorption bands. However, this effect is very small when not measured at the red absorption edge. Hence, it cannot explain the relative amplitude of the 8 ps transient observed at all wavelengths.

(b) Rotational diffusion of excited molecules can lead to changes of the transient absorption on the 10 ps to 500 ps time scale [51]. This effect can, however, not contribute in the magic-angle polarization setup employed in the present experiments.

(c) As shown in table 1 the $S_{3}$ state is of symmetry $A_{g}$ and thus radiative transitions to the $A_{g}$ ground state are of forbidden nature. The present INDO/S CI calculations, which take into account singly and doubly excited configurations, place this state $5000 \mathrm{~cm}^{-1}$ above the $\mathrm{S}_{2}$ level, as do other calculations $[18,19,28]$. INDO/S all-valence electron calculations involving triply and quadruply excited configurations (not available so far) are expected to shift the $A_{g}$ state to lower energies, but presumably not below the $S_{2}\left(B_{2 u}\right)$ state at $30900 \mathrm{~cm}^{-1}$. Consequently, the $A_{g}$ state is not an intermediate in the deactivation cascade when irradiating into the $S_{2}\left(B_{2 u}\right)$ state.

(d) Probably the most tempting interpretation is the assignment of the fast transient absorption to $\mathrm{S}_{2} \rightarrow \mathrm{S}_{n}$ transitions. According to the INDO/S calculation $S_{1} \rightarrow S_{n}$ and $S_{2} \rightarrow S_{n}$ absorption of similar strength are expected in the same spectral region. An argument against this interpretation seems to be the missing $S_{2} \rightarrow S_{0}$ fluorescence. Application of the Strickler-Berg relation to the $S_{0} \rightarrow S_{2}$ absorption band yields a radiative lifetime of 16 ns for the $S_{2}$ state [52]. Hence the measured lifetime of a 8 ps would correspond to a quantum yield of $\mathrm{S}_{2} \rightarrow \mathrm{S}_{0}$ fluorescence of $5 \times 10^{-4}$. However, very recently an upper limit of $\Phi_{2} \leqslant 3 \times 10^{-6}$ for this quantum yield was obtained [52]. As Nickel has pointed out to us (see ref. [52]), this discrepancy can be explained by the fact, that the true radiative lifetime of $S_{2}$ cannot be calculated from the $S_{0} \rightarrow S_{2}$ absorption band by application of the Strickler-Berg relation, since the $S_{1}-S_{2}$ energy difference is only $3300 \mathrm{~cm}^{-1}$ and the statistical limit is not reached [53]. In this case each vibronic level of the $\mathrm{S}_{2}$ state mixes strongly with a large number $(N-1)$ of vibronic levels of the $S_{1}$ state. The oscillator strength of the transitions from the ground state to each of these mixed states is then reduced by a factor of $1 / N$. As a consequence the fluorescence quantum yield is reduced by a factor of $1 / N$. Such a $S_{1}-S_{2}$ level mixing is known for naphthalene [54-58], and the corresponding lengthening of the average radiative lifetime has been explicitly postulated [59]. For biphenylene detailed high-resolution investigations of the $S_{0} \rightarrow S_{2}$ absorption band are not known to us, but there are two indications on $S_{1} \rightarrow S_{2}$ interaction in the literature: Hochstrasser and McAlpine [22] mention the observation of a few sharp lines in the $S_{2}$ absorption band of biphenylene in a biphenyl host crystal, and Zanon [60] reports the observation of sharp lines in the $S_{2}$ absorption band of biphenylene vapor. Thus one may expect for $S_{2}$ of biphenylene a behavior similar to that of $S_{2}$ of naphthalene. In conclusion, the rather long lifetime of $\tau=8$ ps of the fast component of the transient absorption and the absence of an $S_{2} \rightarrow S_{0}$ fluorescence are no arguments against the assignment of the fast component to $S_{2} \rightarrow S_{n}$ absorption.

\section{Summary}

The results of the present investigation can be summarized as follows:

Streak camera measurements of the fluorescence and transient absorption measurements yield a lifetime of $250 \mathrm{ps}$ for the $S_{1}$ state of biphenylene. In transient absorption a component with 8 ps was observed; on the basis of the available data it is assigned to $\mathrm{S}_{2} \rightarrow \mathrm{S}_{n}$ absorption. INDO/S calculations of the $\mathrm{S}_{0} \rightarrow \mathrm{S}_{n}$ spectrum are in good agreement with the experimental data. The calculated $S_{1} \rightarrow S_{n}$ and $S_{2} \rightarrow S_{n}$ spectra predict strong long-axis polarized transitions in the spectral region where transient absorption is observed.

Neither the fluorescence lifetime nor the fluorescence quantum yield $\left(\Phi_{\mathrm{F}}=2.3 \times 10^{-4}\right)$ show a $\mathrm{H} / \mathrm{D}$ isotope effect. The major decay channel of $S_{1}$ is internal conversion $\left(k_{\mathrm{IC}} \approx 4 \times 10^{9} \mathrm{~s}^{-1}\right)$, and the active vi- 
bration is not a $\mathrm{C}-\mathrm{H}$ mode but a mode of the carbon skeleton. The bond orders calculated from INDO/S wavefunctions for the $S_{0}$ and $S_{1}$ states of biphenylene and of cyclobutadiene (see table 2) predict drastic changes of the bond lengths within the four-membered ring from the $S_{0}$ rectangular in direction to a $S_{1}$ square structure. This suggests that the corresponding normal modes are most active in internal conversion. The situation is completely different for aromatic compounds as exemplified by triphenylene and benzene: only minor changes of the bond orders are calculated for their $S_{0} \rightarrow S_{1}$ excitation.

\section{Acknowledgement}

$\mathrm{BD}, \mathrm{MN}$ and $\mathrm{WL}$ greatly appreciate the financial support by the Deutsche Forschungsgemeinschaft, Bonn (SFB 93 "Photochemie mit Lasern"). The authors are greatly indebted to Dr. B. Nickel for critically commenting on the manuscript and for valuable suggestions; they thank Dipl. Chem. D. Luckhaus for PPP calculations and for helpful discussions and $\mathrm{Mr}$. K. Rauch for his competent assistance with preparative work. Thanks are also due to the Hadland Photonics Company, Bovington, for the loan of the streak camera.

\section{References}

[1] E. Hückel, Z. Physik 76 (1931) 628; Z. Elektrochemie Angew. Physik. Chem. 43 (1937) 752, 827; 61 (1957) 866.

[2] R. Breslow, Accounts Chem. Res. 6 (1973) 393.

[3] J.B. Birks, Photophysics of aromatic molecules (Wiley-Interscience, New York, 1970).

[4] J.B. Birks, Organic molecular photophysics (Wiley-Interscience, New York, 1975).

[5] J. Wirz, in: Excited states in organic chemistry and biochemistry, eds. B. Pullman and H. Goldblum (Reidel, Dordrecht, 1977) p. 284.

[6] C.Y. Lin and A. Krantz, J. Chem. Soc. Chem. Commun. (1972) 1111.

[7] S. Masamune, M. Suda, H. Ona and L.M. Leichter, J. Chem. Soc. Chem. Commun. (1972) 1268.

[8] O.L. Chapman, C.L. McIntosh and J. Pacansky, J. Am. Chem. Soc. 95 (1973) 614.

[9] M.P. Cava and M.J. Mitchell, Cyclobutadiene and related compounds (Academic Press, New York, 1967).

[10] G. Maier, Angew. Chem. Intern. Ed. Engl. 13 (1974) 425; 27 (1988) 309.
[11] T. Bally and S. Masamune, Tetrahedron 36 (1980) 343.

[12] G. Maier, S. Pfriem, U. Schäfer and R. Matusch, Angew. Chem. Intern. Ed. Engl. 17 (1978) 520.

[13] J.W. Barton, in: Nonbenzoid aromatics, Vol. 1, ed. J.P. Snyder (Academic Press, New York, 1969);

W. Baker and J.F.W. McOmie, in: Non-benzenoid aromatic compounds, ed. D. Ginsburg (Wiley-Interscience, New York, 1959).

[14] W.C. Lothrop, J. Am. Chem. Soc. 63 (1941) 1187.

[15] E.P. Carr, L.W. Pickett and D. Voris, J. Am. Chem. Soc. 63 (1941) 3231.

[16] R.M. Hochstrasser, Can. J. Chem. 39 (1961) 765.

[17] R.M. Hochstrasser, J. Chem. Phys. 33 (1960) 950.

[18] T. Hoshi, H. Yamamoto, T. Miyauchi, S. Mori, M. Kobayashi and Y. Tanizaki, Ber. Bunsenges. Physik. Chem. 86 (1982) 330.

[19] H. Yamaguchi, M. Ata, J.F.W. McOmie, J.W. Barton and H. Baumann, J. Chem. Soc. Faraday Trans II 79 (1983) 599.

[20] I.W. Hilpern, Trans. Faraday Soc. 61 (1965) 605.

[21 ] J.B. Birks, J.M. de C. Conte and G. Walker, Phys. Letters 19 (1965) 125.

[22] R.M. Hochstrasser and R.D. McAlpine, J. Chem. Phys. 44 (1966) 3325 .

[23] H. Shizuka, T. Ogiwara, S. Cho and T. Morita, Chem. Phys. Letters 42 (1976) 311.

[24] N. Otha, M. Fujita, H. Baba and H. Shizuka, Chem. Phys. 47 (1980) 389.

[25] H.B. Lin and M. Topp, Chem. Phys. Letters 64 (1979) 452.

[26] P.M. Rentzepis and C.J. Mitschele, Anal. Chem. 42 No. 14 (1970) 20A; P.M. Rentzepis, Science 169 (1970) 239.

[27] C. Tetreau, D. Lavalette, E.J. Land and F. Peradejordi, Chem. Phys. Letters 17 (1972) 245.

[28] F. Peradejordi, R. Domingo and J.I. Fernandez-Alonso, Intern. J. Quantum Chem. 3 (1969) 683.

[29] J. Koutecky, J. Chem. Phys. 47 (1967) 1501.

[30] J. Spanget-Larsen, Croat. Chem. Acta 59 (1986) 711.

[31] J.C. Rayez, J.J. Dannenberg, E. Kassab and E.M. Evleth, J. Mol. Struct. 68 (1980) 235.

[32] B. Dick and B. Nickel, Chem. Phys. 78 (1983) 1.

[33] F.M. Logullo, A.H. Seitz and L. Friedman, Org. Synth. Coll. 5 (1973) 54.

[34] B. Lunelli and C. Pecile, Can. J. Chem. 46 (1968) 391.

[35] M. Mardelli and J. Olmstedt, J. Photochem. 7 (1977) 277.

[36] R.S. Craxton, IEEE J. Quantum Electron. QE-17 (1981) 1771.

[37] T. Elsaesser, H.J. Polland, A. Seilmeier and W. Kaiser, IEEE J. Quantum Electron QE-20 (1984) 191.

[ 38 ] H.J. Polland, T. Elsaesser, A. Seilmeier, W. Kaiser, M. Kussler, N.J. Marx, B. Sens and K.H. Drexhage, Appl. Phys. B 32 (1983) 53;

T. Elsaesser and F. Laermer, unpublished results.

[39] A. Seilmeier, K. Spanner, A. Laubereau and W. Kaiser, Opt. Commun. 24 (1978) 237.

[40] T. Elsaesser and W. Kaiser, Chem. Phys. Letters 128 (1986) 231. 
[41] S.J. Strickler and R.A. Berg, J. Chem. Phys. 37 (1962) 814.

[42] B. Dick and G. Hohlneicher, Theoret. Chim. Acta (Berlin) 53 (1979) 221.

[43] R.L. Ellis, G. Kuehnlenz and H.H. Jaffé, Theoret. Chim. Acta (Berlin) 26 (1972) 131.

[44] I. Cohen, J. Chem. Phys. 57 (1972) 5076.

[45] B. Dick and H.-J. Freund, Intern. J. Quantum Chem. 24 (1983) 747 .

[46] N.J. Turro, Modern molecular photochemistry (Benjamin Cummings, Menlo Park, 1978) p. 184.

[47] A. Freiberg, T. Tamm and K. Timpmann, Laser Chem. 3 (1983) 249.

[48] C. Rullière, A. Declemy and P. Kottis, Chem. Phys. Letters 110 (1984) 308.

[49] A.M. Weiner and E.P. Ippen, Chem. Phys. Letters 114 (1985) 456.

[50] N.H. Gottfried, A. Seilmeier and W. Kaiser, Chem. Phys. Letters 111 (1984) 326.
[51] F. Perrin, Acta Phys. Polon. 5 (1936) 335.

[52] B. Nickel and J. Hertzberg, to be published.

[53] A. Amirav, M. Sonnenschein and J. Jortner, J. Phys. Chem. 88 (1984) 5593.

[54] D.S. McClure, J. Chem. Phys. 22 (1954) 1688; 24 (1956) 1.

[55] J. Wessel and D.S. McClure, Mol. Cryst. Liquid Cryst. 58 (1980) 121.

[56] G.W. Robinson and C.A. Langhoff, Chem. Phys. 5 (1974) 1.

[57] C.A. Langhoff and G.W. Robinson, Chem. Phys. 6 (1974) 34.

[58 ] S.M. Beck, D.E. Powers, J.B. Hopkins and R.E. Smalley, J. Chem. Phys. 73 (1980) 2019.

[59] A. Nitzan, J. Jortner and P.M. Rentzepis, Proc. Roy. Soc. A 327 (1972).

[60] I. Zanon, J. Chem. Soc. Faraday II 69 (1973) 1164. 